\title{
Correlative Cryo-electron Tomography to Study Native Protein Organization and Membrane Architecture in Cells
}

\author{
Patrick Hoffmann
}

MRC Laboratory of Molecular Biology, Cambridge, England, United Kingdom

Cellular cryo-electron tomography (ET) can be used to study the organization of proteins and membranes in their native environment. The recent advances of direct electron detection, image processing and cryosample preparation allow for the visualization of the cellular environment at unprecedented molecular detail [1]. We combined these advances with correlative microscopy to study contact sites between the plasma membrane and the endoplasmic reticulum membrane in yeast cells. Such membrane contact sites allow the exchange of small molecules between organelles and they are formed by a set of specific proteins. Using ET and methods, we addressed open questions about the contact site architecture.

Most cellular samples are too thick to image directly by transmission electron microscopy and thus require thinning to electron transparency. Cryo-samples are thinned either by cutting sections from a larger vitrified sample, so called vitreous sectioning [2], or milling of the vitrified sample to lamellae by means of a focused-ion beam (FIB) microscope [3]. Both methods require diligent practice, but can provide direct insights in the protein structure in cells and tissues [1,4]. I will discuss how cryo-FIB sample preparation, which is free of sectioning artefacts, is particularly suited to study the native organization of membrane contact site proteins in yeast cells [5]. Current limitations of cellular cryo-ET in general are low throughput and sample preservation. Our ongoing efforts to image multicellular, complex tissue samples by cryo-ET involve developing novel methodical concepts, and harbor great potential for providing new biological insights.

To directly identify structures of interest within electron tomograms of cells can be challenging. We use yeast genetics and cryo-fluorescence microscopy (FM), to locate fluorescently labeled structures of interest prior to cryo-ET acquisition, and later to identify these structures within the tomographic volume. Furthermore, cryo-FM can help to target the lamella preparation to specific areas on the EM grids or to assess the quality and the attachment of vitreous sections for subsequent cryo-ET [6, 7]. Ultimately, these efforts allowed us to perform 2D class averaging of subvolumes, revealing the supramolecular arrangement of proteins at membrane contact sites. Our results allowed us propose a model on how specific contact site proteins engage with the membranes and how their structural organisation could be implicated in transfer of lipid molecules between the two membranes [5].
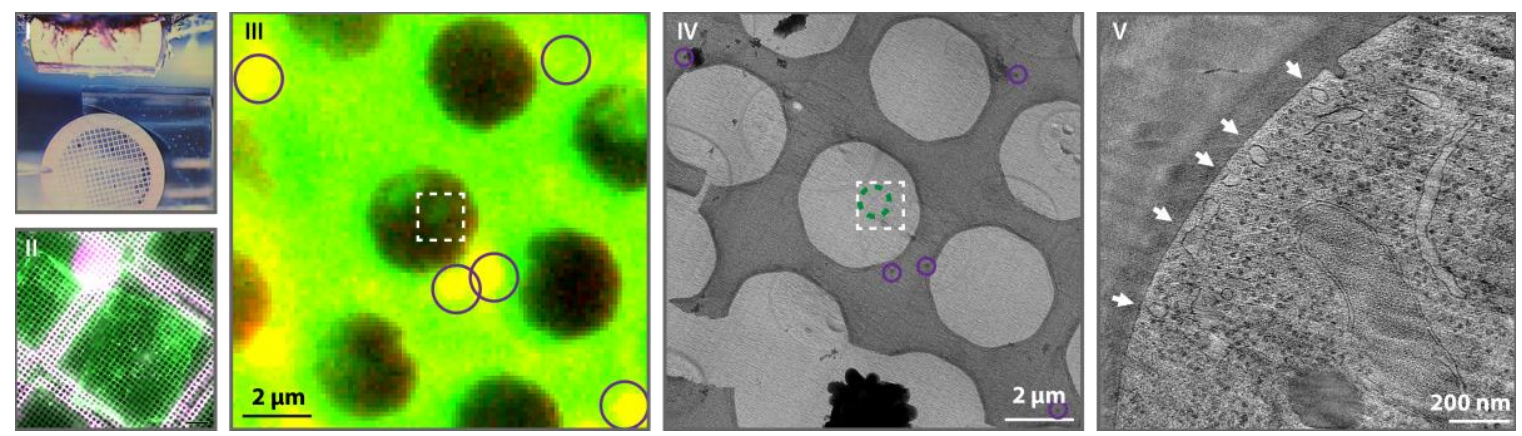

Figure 1. Correlative cryo-ET of vitreous sections of yeast cells. Overview of the setup during vitreous sectioning (I) and cryo-FM image of an individual section on the grid (II). Zoom into a cryo-FM image (III) and the corresponding area seen by TEM (IV). Purple circles highlight fluorescent fiducial markers 
on the carbon film. White box highlights the area of cryo-ET acquisition with fluorescent signal of a membrane contact site protein. Slice through cryo-tomogram (V). Contact sites are indicated by white arrows.

a

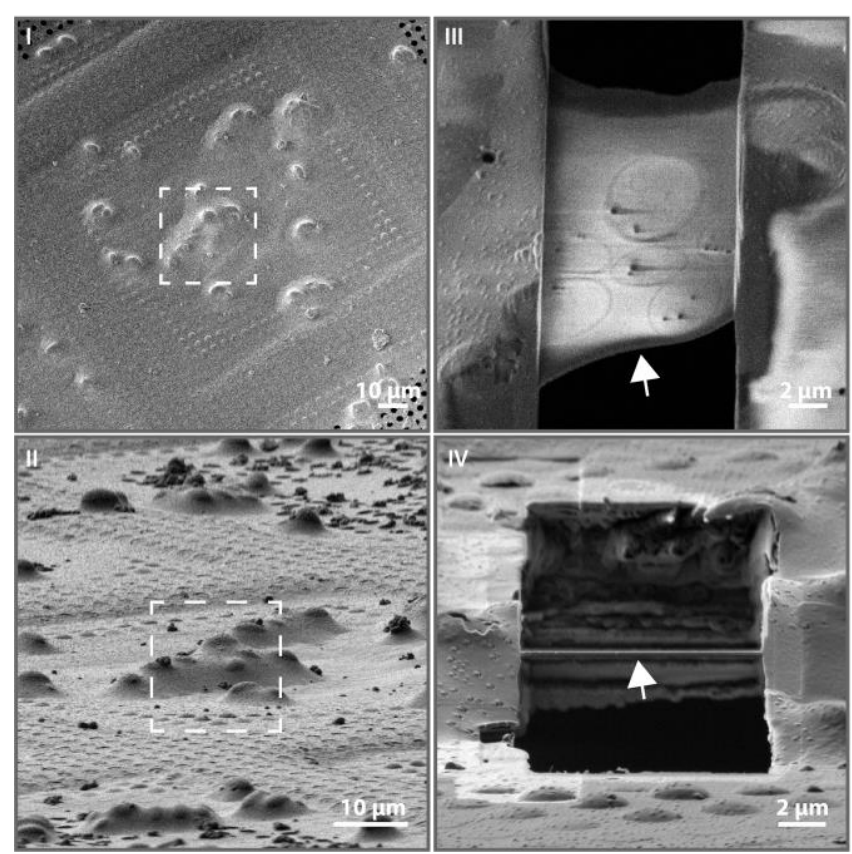

b

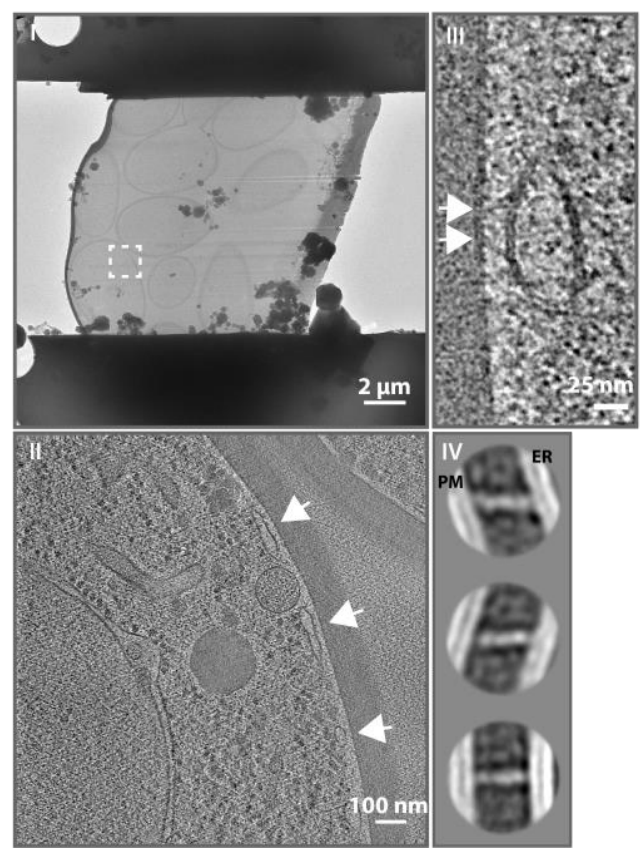

Figure 2. a) Cryo-lamella preparation of yeast by FIB/SEM dual beam microscope. Top view SEM (I) image and front view FIB (II) image of grid square with a group of plunge-frozen yeast cells (white box). SEM (III) and FIB (IV) image of the same group of cells after lamella preparation. White arrows indicate the front of the lamella. b) Cryo-ET of yeast lamella and 2D averaging of protein densities at membrane contact sites. TEM overview of yeast lamella (I). Slice through cryo-tomogram (II). Contact sites are indicated by white arrows. Close-up of contact site. Bridging densities are highlighted by white arrows (III). 2D class averages of protein densities in yeast cells overexpressing the tethering protein tricalbin-3 (IV).

\section{References}

[1] J. Mahamid et al., Science, 351 (2016) 969-972.

[2] J. Dubochet et al., Cryotechniques in Biological Electron Microscopy, Springer, Berlin, 1987.

[3] M. Marko et al., Nat. Methods, 4 (2007) 215-217.

[4] A. Al-Amoudi et al., Nature, 450 (2007) 832-837.

[5] P.C. Hoffmann et al., Dev. Cell, 51 (2019) 488-502.e8.

[6] N. R. Ader et al., eLife, 8 (2019) 303.

[7] T.A.M. Bharat et al., Structure, 26 (2018) 879-886.e3. 